\title{
TACE in colorectal liver metastases - different outcomes in right-sided and left-sided primary tumour location
}

\author{
S Seidl, P Bischoff, A Schaefer, M Esser, V Janzen and A Kovács* \\ Department of Diagnostic and Interventional Radiology and Neuroradiology, MediClin Robert Janker Klinik, Germany
}

\begin{abstract}
Purpose: This study investigated the oncologic outcome following transarterial chemoembolization (TACE) with irinotecan loaded spheres in patients with liver metastases of adenocarcinomas of right-, respectively left-sided colorectal origin (RSCC, LSCRC).

Materials and methods: 21 Patients (pts) with unresectable mono- or bilobar colorectal liver metastases (CRLM), liver-only or liver-dominant metastatic spread, with less than $25 \%$ of the liver parenchyma involved and progression after second line systemic chemotherapy underwent lobar irinotecan TACE. Tolerability, safety and oncologic outcome were assessed in terms of intervention-associated side effects, respectively local tumour control (LTC), progression free survival (PFS) and overall survival ( $\mathrm{mOS})$.

Results: 16 pts with left-sided and 5 with right-sided colonic primary with a mean volumetric tumour burden of $5.27 \pm 6.26 \%$, median $2.89 \%$ (range $0.23-24.1 \%$ ) received in total $49 \mathrm{TACE}$, in average 2.33 interventions per patient. Treatment-related abdominal pain occurred in $4.08 \%$ on the day of intervention and could sufficiently be controlled with pain medication on demand. LTC (CR, PR or SD) in the liver was achieved in 20/21 (95.2\%), 19/21 (90.4\%) and 16/21 (76.2\%) patients at 1, 3 and 6 months, respectively. PFS was 5.5 mo in LSCRC, respectively 3.75 mo in RSCC. mOS was 33 mo after the first TACE in LSCRC, respectively 17 mo in RSCC.
\end{abstract}

Conclusion: TACE with irinotecan loaded spheres is a safe and well tolerated procedure in the treatment of CRLM. The promising results in terms of mOS especially in tumours of left-sided origin deserve further investigation in larger prospective trials.

\section{Introduction}

In patients with liver metastases of colorectal cancer (CRC) the key goals for improving outcome include early detection, identification of patients at high risk for recurrence and prognostic indicators of treatment response [1]. Tailored multidisciplinary approaches, combining systemic and locoregional therapies enable to maximize efficacy, to decrease intrahepatic recurrence and to minimize toxicity. The advent of new drugs (Irinotecan and Oxaliplatin) and targeted therapies (Bevacizumab, Cetuximab and Panitumab) present the opportunity for a personalized approach to the patient [2]. Attributed to the improved systemic therapy the median overall survival (mOS) has already risen from about 12 months (mo) in the mid nineties to 24 mo recently [3]. Although regarded to be not curative, Transarterial Chemoembolization (TACE) is a valuable adjunct to surgery, regarding pre- and post-operative downsizing, conversion to resectability as well as improvement of recurrence rate after liver resection [4]. The recent concept of oligometastatic disease and the implementation of the toolbox of locoregional therapies led to a radical rethinking of treatment options in CRLM [3]. Patients with an oligometastatic disease and with a less aggressive tumour biology may entail therapeutic potentials beyond surgery and CTX alone. In the endovascular therapy of CRLM the administration of the drug, particularly Irinotecan plays a decive role. One area which has emerged as an important factor of predicting treatment outcome in CRLM is the primary tumour location [5]. Right-sided colon cancer (RSCC) and left-sided colorectal cancer (LSCRC) differ with respect to their biology, genomic patterns, molecular profile, metastatic pathways and ultimately immunogenicity [6]. Resulting from this difference, certain therapies already proved efficacy predominantly in one subgroup, according to this selective internal radiotherapy (SIRT) performed better in RSCC and conversely Cetuximab, an anti-EGFR monoclonal antibody better in LSCRC [68]. For the development of effective therapy regimes and avoidance of ineffective but onerous treatments, it is essential to evaluate RSCC and LSCRC tumours as separate primaries, and design the therapy regime considering the differences between these tumours [9].

The herein presented study on TACE in CRLM of primarily rightsided and left-sided CRC is to our best knowledge the first analysis on this issue.

\section{Materials and methods}

\section{Study design}

The retrospective analysis presented herein was designed to evaluate the safety and efficacy of Irinotecan-TACE (Transarterial Chemoembolization using loaded spheric particles) in the treatment of colorectal liver metastases of right-sided, respectively left-sided

*Correspondence to: Attila Kovács, Department of Diagnostic and Interventional Radiology and Neuroradiology, MediClin Robert Janker Klinik, Villenstraße 8, 53129 Bonn, Germany, Tel: +49 2285306 - 501, Fax +49228 5306 - 502, E-mail: attila.kovacs@mediclin.de

Key words: colorectal liver metastases, hepatic artery infusion, transarterial chemoembolization, right-sided colorectal cancer, left-sided colorectal cancer

Received: January 10, 2020; Accepted: January 27, 2020; Published: January 30, 2020 
colonic origin. Institutional Review Board approval was obtained, and all patients provided informed consent. The study was conducted in accordance with the European Union regulations, the Declaration of Helsinki, and the IHC Harmonized Tripartidute Guideline for Good Clinical Practice (GCP).

\section{Patient inclusion criteria}

Patients aged $>18$ years with histologically proven uni- or bilobar unresectable colorectal liver metastases who showed progression after second line systemic chemotherapy were considered eligible for the study. Patients were required to have liver-only or liver-dominant metastatic spread, with less than $25 \%$ of the liver parenchyma involved by tumour, further ECOG-status 0-1 (Eastern Cooperative Oncology Group). Patients with both synchronous and metachronous metastases were included as well as patients with both tumour location from the coecum, ascending and transverse colon (RSCC), respectively from the descending colon, sigma and rectum (LSCRC).

\section{Exclusion criteria}

Patients with tumour invasion of the portal vein or the main biliary duct, portal vein thrombosis, serum bilirubin level higher than $2 \mathrm{mg} / \mathrm{dl}$, transaminase values greater than three times upper limit of normal (ULN), venoocclusive disease after Oxaliplatin-therapy, renal insufficiency, contrast agent allergy and contraindications for Irinotecan-administration, were excluded.

\section{Treatment protocol and imaging studies}

Endovascular therapy was performed alternating with the systemic chemotherapy. The latter was interrupted for at least one week before starting the endovascular treatment. Endovascular treatment was always performed segmental or lobar. Treatments were repeated in 4-week intervals until complete remission was achieved in the target area, however at most up to 4 times. In bilobar disease treatment was started with the lobe with the greater disease involvement.

Pretreatment MRI images were analyzed to evaluate the number, size, location of the metastases and to calculate the volumetric tumour burden. TACE was performed in a dedicated angio suite with ConeBeam CT (CBCT) under local anaesthesia. As CRC liver metastase are predominantly intermediate or hypo-vascularized and usually occult in standard angio runs a dedicated 3-phasic injection protocol was used to achieve both a capillary enhancement, that occurs at least 25-30 sec after injection as well as the feeding vessels of the target lesion.[10] This injection protocol used in the subselective position was as follows: first $15 \mathrm{cc}$ of contrast were injected with a flowrate of $1 \mathrm{cc} / \mathrm{sec}$, followed by a break of $10 \mathrm{sec}$ and than $10 \mathrm{cc}$ of contrast were injected with $3 \mathrm{cc} / \mathrm{sec}$ followed by $10 \mathrm{cc}$ of saline at $3 \mathrm{cc} / \mathrm{sec}$. Breathold was started $20 \mathrm{sec}$ after the first injection.

Standard periinterventional medication consisted of cortisone and a single-shot antibiotic prophylaxis (500 mg Ciprofloxacin i.v.). In case of suspected infection antibiotic therapy was continued for two days via i.v. line and further 5 days orally. Treatment related nusea and pain were treated with Ondansetron $4 \mathrm{mg} /$ day and Ibuprofen $400 \mathrm{mg}$, each three times a day. Additionally Piritramid ( $15 \mathrm{mg}$ via i.v. perfusor) was available for the patients on demand.

In each therapy session one vial of spheric embolic agents HepaSpheres $^{\circ}$ (Merit), preloaded with $75 \mathrm{mg}$ of Irinotecan according to the manufacturer's instructions were administered from the intended treatment position. Each vial of preloaded spheres was mixed gently with $10 \mathrm{cc}$ of contrast and $10 \mathrm{cc}$ of acqua until a homogenous suspension was achieved. The latter mixture was applied very slowly via microcatheter until a substasis was achieved.

After catheter removal femoral access closure was achieved with manual compression. The first day after procedure blood values were controlled and the second day after procedure patients underwent contrast enhanced MRI scan to assess for complications and early results of the treatment.

\section{Study endpoints}

Primary objectives of the study were to investigate treatment tolerability during the first two days following the therapy, treatment safety during the first four weeks. Local tumour control was assessed in intervals of 2 months by multiparametric MR imaging of the liver. Oncologic outcome was evaluated in terms of median overall survival (mOS) and progression free survival (PFS). Adverse events (AE) were defined as any undesirable experience beyond the usual postembolization syndrome (PES; temporary flu-like symptoms, nausea and epigastric sensation of pressure, those commonly resolve the day after intervention) comprising pain, vomiting, abnormal laboratory value. Severe adverse events (SAE) included inpatient hospitalization, events that required medical or surgical intervention, life-threatening adverse experiences up to death. AE intensity was graded according to the National Institute of Health (NIH-CTC) criteria V 4.0 (www.ctep. cancer.gov). Local tumour control was defined as complete response, partial response or stable disease according to the RECIST criteria, version 1.1 [11]. Response to treatment was evaluated on the basis of the multiparametric MRI of the liver, including multiplanar ce T1w, transversal T2w fs and transversal DWI scans at designated time points after the designated end point following the individual course of TACE-treatments (including 1 to 4 TACE-sessions, each perfomed in intervals of four weeks) after 1, 2 and 6 month. Staging with thoracal and abdominopelvic CT scans were performed every 3 months to assess extrahepatic disease. In case of hepatic tumour recurrence at an already finalized (up to 4 cycles of TACE) treatment site, patients were redirected to systemic therapy. The same procedure was applied for de novo liver metastases during the course of the study. Secondary endpoints were overall survival and liver-specific disease progression.

\section{Statistical analysis}

Descriptive statistics are used to summarize patient baseline characteristics. The rate of AE, respectively SAE is decribed in relation to the number of DEBIRI-treatments performed. Local tumour control rates, defined as complete response, partial response and stable disease are reported in relation to the number of study patients. Kaplan-Meier analyses were performed to estimate liver-specific progression-free survival (PFS) and overall survival (OS). Statistical analyses were performed with Statistical Analysis Software (SAS), version 9.2 (SAS Institute Inc., Cary, North Carolina).

\section{Results}

\section{Patients and procedures}

From January 2013 to October 2015 twentyone patients (11 males, 10 females, mean age $62.5 \pm 13.2$ years; median 68 years; range $35-85$ years) with colorectal liver metastases were enrolled in the study. The follow-up period ended in April 2017. 16 pts had a left-sided primary (LSCRC), among them 5 pts with RAS-mutation ( $2 \mathrm{~N}$ - and K-RAS, respectively 3 K-RAS). 5 pts had a right-sided primary (RSCC), among them one with K-RAS-mutation. Primary cancers were in all 21 pts adenocarcinomas, in 13 pts moderately and in 8 pts low differentiated. 
All 21 pts had first and second line systemic therapy prior to the first Irinotecan-TACE. 7 pts had synchronous and 14 pts metachronous liver metastases. 18 pts had only liver metastases, 3 pts had liver-dominant disease with additional, small and not life threatening lung metastases. Mean volumetric tumour burden in the liver was $5.27 \pm 6.26 \%$, median $2.89 \%$ (range $0.23-24.1 \%$ ). The mean interval between detection of the liver metastases and first TACE were 10.7 months. In total 49 TACE were performed, equivalent to 2.33 TACE per patient in average. During the course of the study 6 patients $(28.6 \%)$ were redirected to systemic chemotherapy, 4 due to intrahepatic recurrence, respectively 2 due to de novo liver metastases.

\section{Tolerability and safety}

Treatment-related side-effects beyond the usual post-embolization syndrome were rare. Pro re nata medication with Piritramid for abdominal pain control on the same day was required in 2 of 49 TACE-procedures $(4.08 \%)$, but in the same patient. Postinterventional abdominal discomfort and nausea was sufficiently treated with Ibuprofen, respectively Ondansetron. Owed to the fact, that the therapeutic approach presented herein was better tolerated as initially expected, routine pain and antiemetic medication were turned into medication on demand during the course of the study. In none of the patients occurred SAEs (Figure 1).

\section{Oncologic outcome and survival analysis}

Local tumour control (complete response, partial response or stable disease) in the liver was achieved in 20/21 (95.2\%), 19/21 (90.4\%) and $16 / 21(76.2 \%)$ patients at 1,3 and 6 months, respectively. $2 / 3$ patients with concomitant lung metastases showed a pulmonary response to the liver-directed therapy, both with shrinking of the lung metastases each less than $25 \%$. PFS was 5.5 mo in LSCRC, respectively 3.75 mo in RSCC. mOS was 33 mo after the first TACE in LSCRC, respectively 17 mo in RSCC (Figure 2). RAS-mutational status had no impact on mOS and PFS.

\section{Discussion}

The Irinotecan-TACE presented herein, revealed a good tolerability and safety. During the course of the study less pain medication was required than initially supposed. SAE were not reported, only occasionally minor AE. Prognostic indicators of treatment response were the location of the primary in RSCC, respectively LSCRC, further the hepatic tumour burden as well as the load of therapeutic drugs administered before the endovascular therapy. Our data suggest, that patients with CRLM of left-sided origin benefit significantly more from Irinotecan-TACE as patients with right-sided origin do. Predictors of a beneficial outcome were as follows: a low metastatic burden in the liver, a low mean CA 19-9 level, as well as a low systemic Irinotecan, respectively Capecitabine load (Table 1).

Colorectal cancer is the third most common cancer worldwide, with one million new cases each year [12]. It is the second leading cause of cancer death in Western Countries [13]. Up to $60 \%$ of the patients with CRC develop metastases during the course of their disease, at which point survival rates drop markedly [12]. The liver is the most common site of CRC-metastasis due to its anatomical linkage to the portal circulation [13]. If untreated, the median survival of patients with hepatic metastases (CRLM) is generally 6-7 month, not exceeding 12 months [14]. In the presence of CRLM hepatic resection is considered the best chance for long-term survival (5-year survival up to $40 \%$ ), but only $10 \%$ to $25 \%$ of the patients can be resected with curative intention [14]. In metastatic CRC, the liver dominance and the tumour burden

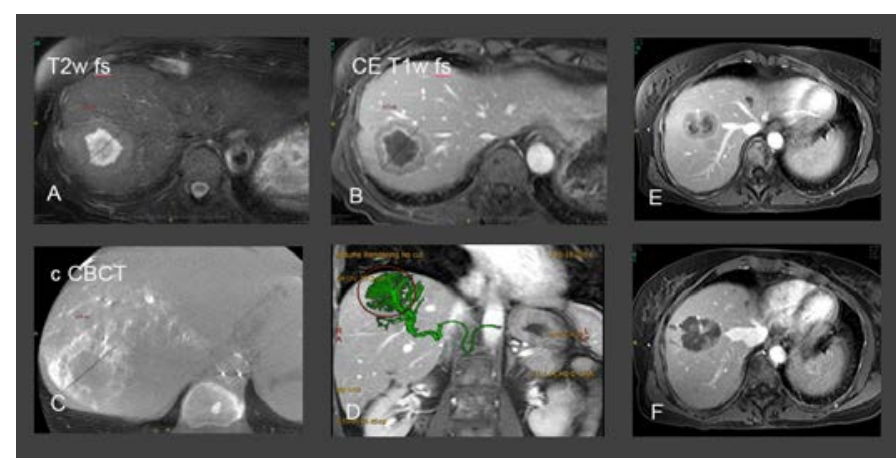

Figure 1. A-B. Preinterventional MRI for treatment planning. C-D: CBCT and fusion of MRI- and CBCT-images for treatment guidance. MRI and CBCT images were superimposed (D) to ensure the full coverage of the target lesion. E-F: Preinterventional ceT1w image of another target and postinterventional follow up image performed 48 after treatment proving the effective devascularization of the metastasis

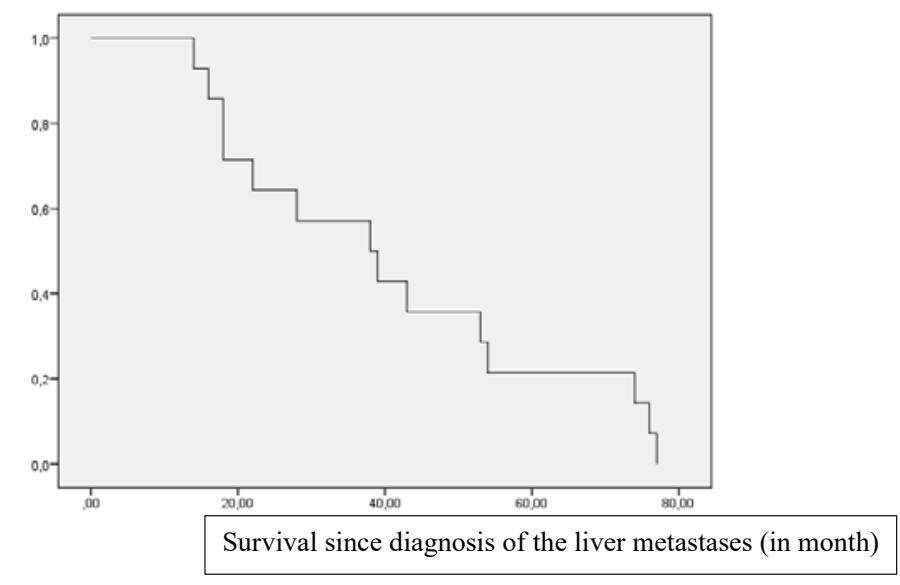

Figure 2. Overall survival in a series of 21 patients with liver-only or liver-dominant colorectal metastases treated with sequential Irinotecan-TACE, after failure of first- and second-linde systemic chemotherapy

Table 1: The comparison of the characteristics in the five patients with the best survival vs the five patients with the worst survival revealed further prognostic parameter.

\begin{tabular}{|c|c|c|}
\hline & Best 5 & Worst 5 \\
\hline mOS & $35.4 \mathrm{mo}$ & $9.5 \mathrm{mo}$ \\
\hline mean tumour burden & $3 \%$ & $11 \%$ \\
\hline mean CA 19-9 level & $<10 \mathrm{kU} / 1$ & $>10 \mathrm{kU} / 1$ \\
\hline mean systemic Irinotecan load & $<950 \mathrm{mg} / \mathrm{m}^{2}$ & $1285 \mathrm{mg} / \mathrm{m}^{2}$ \\
\hline mean Capecitabine load & $<30.000 \mathrm{mg}$ & $168.000 \mathrm{mg}$ \\
\hline
\end{tabular}

in the liver are predictors of survival [1]. Local tumour control enables prolonged mOS. The preservation of the QoL requires on the one hand local symptom control and then chemo-holidays, patients like to take advantage. The controlled oligometastatic disease enables a prologed life with a good life quality. In this period patients are not compromised in their social every day life and many of them return to professional everyday.

Local ablative therapies (LAT), like radiofreqency (RFA), microwave (MWA) and cryo-ablation (Cryo) are increasingly used to treat unresectable CRLM [1]. The results of the randomized EORT-CLOCC trial argue in favour of a combined therapy of LAT and chemotherapy (CTX) over CTX alone. This randomized phase II-trial with a median follow-up of 9.7 years on 119 patients with unresectable CRLM revealed a mOS of $35.9 \%$ in the combined arm vs. only $8.9 \%$ in the CTX-arm after eight years [15]. The recent ESMO-guidelines concluded subsequently, that the attempt to eradicate all visible metastatic lesions using the 
best instrument from the toolbox of LATs, in combination with CTX is one of the determining key goals for improving outcome [1,3]. A generally acknowledged limitation of thermal ablations is the size of the target, conterminous that e.g. RFA is limited in lesions larger than $3.5 \mathrm{~cm}$. Both, systemic and localized drug delivery have the potential of downsizing initially nonresectable CRLM. Systemic neoadjuvant chemotherapy was able to downsize $22.5 \%$ of patients (meta-analysis including $1886 \mathrm{pts}$ ), meanwhile transarterial chemoembolization with irinotecan loaded drug eluting beads (DEBIRI-TACE) downsized $40 \%$ of 10 pts assessed [16,17].

The technique of TACE in CRC liver metastases is different from TACE in hepatocellular carcinoma (HCC), synonymic the technique of HCC-TACE is not one to one applicable to TACE in CRLM. In latter the administration of the drug, particularly of Irinotecan plays a decive role, meanwhile in HCC the embolization is of major importance. Irinotecan is a prodrug, that is metabolized by hydrolysis to the appr. 1000-times more potent active metabolite SN-38 in hepatocytes [18]. $\mathrm{SN}-38$ again arrives by osmosis into the targeted tumour cells. The pharmacokinetics of Irinotecan, respectively SN-38 related to DEBIRITACE are quantifiable [17].

The performance of TACE in CRLM is attributed to the pathophysiological peculiarity of secondary liver malignancies in general. Once hepatic metastases grow more than $2 \mathrm{~mm}$ in size, they derive their blood supply from the hepatic artery, while normal hepatocytes are perfused mostly from the portal circulation $[1,4,19]$. This is the general rationale of endovascular treatment in the liver. A special feature of endovascular Irinotecan treatment of the liver is that it requires activation by normal hepatocytes. Activation is accomplished by the enzymes carboxylesterase 1 and 2 (CES-1 and -2) that convert the prodrug Irinotecan to its active form 7-Ethyl-10-hydroxy-camptothecin (SN-38) [17,20-22]. Therefore, in contrast to TACE in the treatment of other cancers, subselective embolization is not recommended in CRLM for two reasons. The first is the fact that Irinotecan requires activation by normal liver parenchyma via hydrolysis. The second reason is the desire to treat all metastatic disease, including radiographic occult lesions, particularly important in intermediate or hypovascularized liver metastases [23]. According to these insights the technical endpoint of TACE in our study was the substasis.

One major limitation of locoregional therapies is an advanced tumour load [1]. Huppert and coworkers demonstrated already 2013 that the limited survival benefit in patients with CRLM in a salvage setting is attributed to the advanced intrahepatic tumour burden [24]. Due to the significantly reduced response rate $(4-15 \%)$ of reinduction therapy with FOLFOX / FOLFIRI after failure of $1^{\text {st }}$ and $2^{\text {nd }}$ line therapy TACE may be better implemented earlier instead of a salvage therapy [25]. However it requires further investigation to assess the optimal timing for endovascular locoregional drug delivery during the treatment of CRLM. Our results support the hypothesis, that some patient subgroups, e.g. pts with LSCRC liver metastases would benefit from the early endovascular therapy. As radioembolization with $\mathrm{Y}^{90}$ failed to prove survival benefit in patients with CRLM of left-sided origin, SIRT should be considered as a liver-directed therapy option in liver metastases of right-sided origin, meandwhile for these patients SIRT proved a survival benefit of 4 months [7].

Several studies proved already safety, tolerability and oncologic response after DEBIRI-TACE. Fiorentini and coworkers revealed in a prospective randomized study a significant PFS- and mOS-benefit under DEBIRI as compared to FOLFIRI (PFS 7 mo vs 4 mo respectively mOS $22 \mathrm{mo}$ vs $15 \mathrm{mo}$ ) [26]. Further a significantly better QoL in the
DEBIRI-arm as compared to FOLFIRI cohorts at 1,3 and 8 months ( $\mathrm{p}$ $=0.038, p=0.025$ and $p=0.025$ respectively). Aliberti et al assessed the efficacy of lobar or segmental DEBIRI in 82 patients whom had failed at least two prior lines of chemotherapy [27]. An OS of 25 months and time to progression (TTP) of 8 months was reported. Further a general improvement in QoL in $90 \%$ of patients that lasted 32 weeks. Martin and coworkers demonstrated in the combined therapy group (DEBIRI + FOLFOX + Bevacizumab) as compared to FOLFOX+Bevacizumab alone a significant improvement in overall response rates (ORR) at 6 month $(\mathrm{p}=0.05)$ and liver PFS $(\mathrm{p}=0.05)$ [17]. Iezzi and coworkers evaluated the safety, tolerance and efficacy of a combined therapy (DEBIRI + Capecitabine) in 20 patients with liver-dominant disease (> $80 \%$ of metastatic burden confined to the liver) and liver metastases refractory to chemotherapy (two or more lines). PFS and OS were 4 and 7.3 months, respectively [28].

Despite the promising oncologic results our study has limitations. The cohort assessed in this single center study is small, a further limitation is the retrospective design of the study.

It is not questionable that in liver only or liver dominant metastatic pattern of CRC the treatment of the liver should be priorized and optimized, in terms of improved efficacy and improved tolerability. Today is not the question if drug delivery should be performed either systemic or localized, but the combination of both to maximize therapeutic efficacy in the liver meanwhile minimizing treatment toxicity through a tailored multidisciplinary approach - eg combination of systemic FOLFOX with liver directed Irinotecan, in LSCRC basically combinable with Cetuximab. Future perspectives encompass a personalized approach focussing on prognostic and predictive factors of the tumour microenvironment, the location of the primary and the immunoscore.

\section{References}

1. Zarour LR, Anand S, Billingsley KG, Bisson WH, Cercek A, et al. (2017) Colorectal Cancer Liver Metastasis: Evolving Paradigms and Future Directions. Cell Mol Gastroenterol Hepatol 3: 163-173. [Crossref]

2. Kulendran M, Stebbing JF, Marks CG, Rockall TA (2011) Predictive and prognostic factors in colorectal cancer: a personalized approach. Cancers (Basel) 3: 1622-1638. [Crossref]

3. Van Cutsem E, Cervantes A, Adam R, Sobrero A, Van Krieken JH, et al. (2016) ESMO consensus guidelines for the management of patients with metastatic colorectal cancer. Ann Oncol 27: 1386-1422. [Crossref]

4. Massmann A, Rodt T, Marquardt S, Seidel R, Thomas K, et al. (2015) Transarterial chemoembolization (TACE) for colorectal liver metastases--current status and critical review. Langenbecks Arch Surg 400: 641-659. [Crossref]

5. Young S, Golzarian J (2018) Primary Tumor Location in Colorectal Cancer: Comparison of Right- and Left-Sided Colorectal Cancer Characteristics for the Interventional Radiologist. Cardiovasc Intervent Radiol 41: 1819-1825. [Crossref]

6. Wang F, Bai L, Liu TS, Yu YY, He MM, et al. (2015) Right-sided colon cancer and left-sided colorectal cancers respond differently to cetuximab. Chin J Cancer 34: 384393. [Crossref]

7. Wasan HS, Gibbs P, Sharma NK, Taieb J, Heinemann V, et al. (2017) First-line selective internal radiotherapy plus chemotherapy versus chemotherapy alone in patients with liver metastases from colorectal cancer (FOXFIRE, SIRFLOX, and FOXFIREGlobal): a combined analysis of three multicentre, randomised, phase 3 trials. Lancet Oncol 18: 1159-1171. [Crossref]

8. Tejpar S, Stintzing S, Ciardiello F, Tabernero J, Van Cutsem E, et al. (2017) Prognostic and Predictive Relevance of Primary Tumor Location in Patients With RAS Wild-Type Metastatic Colorectal Cancer: Retrospective Analyses of the CRYSTAL and FIRE-3 Trials. JAMA Oncol 3: 194-201. [Crossref]

9. Baran B, Mert Ozupek N, Yerli Tetik N, Acar E, Bekcioglu O, et al. (2018) Difference Between Left-Sided and Right-Sided Colorectal Cancer: A Focused Review of Literature. Gastroenterology Res 11: 264-273. [Crossref] 
10. Schernthaner RE, Haroun RR, Duran R, Lee H, Sahu S, et al. (2016) Improved Visibility of Metastatic Disease in the Liver During Intra-Arterial Therapy Using Delayed Arterial Phase Cone-Beam CT. Cardiovasc Intervent Radiol 39: 1429-1437. [Crossref]

11. Eisenhauer EA, Therasse P, Bogaerts J, Schwartz LH, Sargent D, et al. (2009) New response evaluation criteria in solid tumours: revised RECIST guideline (version 1.1). Eur J Cancer 45: 228-247. [Crossref]

12. Fernandez Moro C, Bozoky B, Gerling M (2018) Growth patterns of colorecta cancer liver metastases and their impact on prognosis: a systematic review. BMJ Open Gastroenterol 5: e000217.

13. De Divitiis C, Nasti G, Montano M, Fisichella R, Iaffaioli RV, et al. (2014) Prognostic and predictive response factors in colorectal cancer patients: between hope and reality. World J Gastroenterol 20: 15049-15059. [Crossref]

14. Valderrama-Trevino AI, Barrera-Mera B, Ceballos-Villalva JC, Montalvo-Javé EE (2017) Hepatic Metastasis from Colorectal Cancer. Euroasian J Hepatogastroenterol 7: $166-175$.

15. Meijerink MR, Puijk RS, van Tilborg AAJM, Henningsen KH, Fernandez LG, et al (2018) Radiofrequency and Microwave Ablation Compared to Systemic Chemotherapy and to Partial Hepatectomy in the Treatment of Colorectal Liver Metastases: A Systematic Review and Meta-Analysis. Cardiovasc Intervent Radiol 41: 1189-1204. [Crossref]

16. Ruers T, Van Coevorden F, Punt CJ, Pierie JE, Borel-Rinkes I, et al. (2017) Local Treatment of Unresectable Colorectal Liver Metastases: Results of a Randomized Phase II Trial. J Natl Cancer Inst: 109. [Crossref]

17. Martin RC, 2nd, Scoggins CR, Tomalty D, Schreeder M, Metzger T, et al. (2012) Irinotecan drug-eluting beads in the treatment of chemo-naive unresectable colorectal liver metastasis with concomitant systemic fluorouracil and oxaliplatin: results of pharmacokinetics and phase I trial. J Gastrointest Surg 16: 1531-1538. [Crossref]

18. Liu DM, Thakor AS, Baerlocher M, Alshammari MT, Lim H, et al. (2015) A review of conventional and drug-eluting chemoembolization in the treatment of colorectal liver metastases: principles and proof. Future Oncol 11: 1421-1428. [Crossref]

19. Bacchetti S, Pasqual E, Crozzolo E, Pellarin A, Cagol PP (2009) Intra-arterial hepatic chemotherapy for unresectable colorectal liver metastases: a review of medical devices complications in 3172 patients. Med Devices (Auckl) 2: 31-40. [Crossref]
20. Murono K, Tsuno NH, Kawai K, Sasaki K, Hongo K, et al. (2012) SN-38 overcomes chemoresistance of colorectal cancer cells induced by hypoxia, through HIF1alpha. Anticancer Res 32: 865-872. [Crossref]

21. Lewis AL, Hall B (2019) Toward a better understanding of the mechanism of action for intra-arterial delivery of irinotecan from DC Bead((TM)) (DEBIRI). Future Oncol 15: 2053-2068. [Crossref]

22. Levy EB, Peer C, Sissung TM, Venkatesan A, Pandalai P, et al. (2019) Pilot Study Comparing Systemic and Tissue Pharmacokinetics of Irinotecan and Metabolites after Hepatic Drug-Eluting Chemoembolization. J Vasc Interv Radiol 30: 19-22. [Crossref]

23. Young S, D'Souza D, Flanagan S, Golzarian J (2017) Review of the Clinical Evidence for the Use of DEBIRI in the Treatment of Colorectal Metastatic Disease. Cardiovasc Intervent Radiol 40: 496-501. [Crossref]

24. Huppert P, Wenzel T, Wietholtz H (2014) Transcatheter arterial chemoembolization (TACE) of colorectal cancer liver metastases by irinotecan-eluting microspheres in a salvage patient population. Cardiovasc Intervent Radiol 37: 154-164. [Crossref]

25. Nishiofuku H, Tanaka T, Matsuoka M, Otsuji T, Anai H, et al. (2013) Transcatheter arterial chemoembolization using cisplatin powder mixed with degradable starch microspheres for colorectal liver metastases after FOLFOX failure: results of a phase I/ II study. $J$ Vasc Interv Radiol 24: 56-65. [Crossref]

26. Fiorentini G, Aliberti C, Tilli M, Mulazzani L, Graziano F, et al. (2012) Intra-arterial infusion of irinotecan-loaded drug-eluting beads (DEBIRI) versus intravenous therapy (FOLFIRI) for hepatic metastases from colorectal cancer: final results of a phase III study. Anticancer Res 32: 1387-1395.

27. Aliberti C, Fiorentini G, Muzzio PC, Pomerri F, Tilli M, et al. (2011) Trans-arteria chemoembolization of metastatic colorectal carcinoma to the liver adopting DC Bead(R), drug-eluting bead loaded with irinotecan: results of a phase II clinical study Anticancer Res 31: 4581-4587.

28. Iezzi R, Marsico VA, Guerra A, Cerchiaro E, Cassano A, et al. (2015) Trans-Arterial Chemoembolization with Irinotecan-Loaded Drug-Eluting Beads (DEBIRI) and Capecitabine in Refractory Liver Prevalent Colorectal Metastases: A Phase II SingleCenter Study. Cardiovasc Intervent Radiol 38: 1523-1531. [Crossref]

Copyright: (C2020 Seidl S. This is an open-access article distributed under the terms of the Creative Commons Attribution License, which permits unrestricted use, distribution, and reproduction in any medium, provided the original author and source are credited. 